Am. J. Trop. Med. Hyg. 51(4), 1994. pp. 384-388

Copyright 1994 by The American Society of Tropical Medicine and Hygiene

\title{
DRUG DISCOVERY, DEVELOPMENT AND DEPLOYMENT: A REPORT FROM THE 28th JOINT CONFERENCE OF THE U.S.-JAPAN PARASITIC DISEASES PANELS, BALTIMORE, MARYLAND, JULY 1993*
}

DONALD J. KROGSTAD, MAMORU SUZUKI, CAROLE A. LONG, YOSHIKI AOKI, AKIRA ISHII, AND STEPHANIE L. JAMES

Tulane School of Public Health and Tropical Medicine, New Orleans, Louisiana; Gunma University School of Medicine, Maebashi, Japan; Hahnemann University, Philadelphia, Pennsylvania; Nagasaki University, Nagasaki, Japan; National Institute of Health, Tokyo, Japan; National Institute of Allergy and Infectious Diseases, National Institutes of Health, Bethesda, Maryland

\begin{abstract}
The 28th Joint Conference of the Parasitic Diseases Panels of the U.S.-Japan Cooperative Medical Sciences Program held in Baltimore, Maryland focused on current research within both countries on antiparasitic chemotherapy. This meeting report summarizes presentations of work in progress on antiparasitic drugs currently in use and drugs under development or in clinical trials, as well as reports on potentially unique parasite characteristics that may provide targets for development of future therapeutics.
\end{abstract}

The U.S.-Japan Cooperative Medical Sciences Program was established in 1965 by joint agreement of the President of the United States and the Prime Minister of Japan. The bilateral program was organized to promote cooperative research on health problems of recognized importance in Southeast Asia. Parasitic disease was one of the five original areas selected for collaborative research at that time. Currently, other Panels affiliated with the Program include cholera, leprosy, tuberculosis, viral diseases, hepatitis, malnutrition, environmental mutagenesis and carcinogenesis, and the acquired immunodeficiency syndrome (AIDS). There is also an immunology board intended to provide expertise in this fundamental area of research. The first charter of scientific guidelines to the Parasitic Diseases Panel specified that the research concerns be confined to schistosomiasis and filariasis, mainly due to their important impact on world health and economic development. In 1985 , however, the guidelines were expanded and research on malaria and other vector-borne parasitic diseases was incorporated into the program. Annual conferences are held alternatively in the U.S. and Japan. Participants include members of the U.S. and Japanese Parasitic Diseases Panels

* Editor's footnote: The publication of this meeting report is a departure from the usual format for material appearing in the Journal. Citation of specific information included herein must refer to the original reference. This report is not a citable document. as well as other members of the research community within the U.S., Japan, and other Southeast Asian countries. These meetings are open to the public.

The 28th Joint Conference of the Parasitic Diseases Panels of the U.S.-Japan Cooperative Medical Science Program was held in Baltimore, Maryland July on 19-22, 1993. It covered aspects of the immunology, cell biology, epidemiology, and vector biology of malaria, schistosomiasis, filariasis and trypanosomes. This report summarizes the discussions from a minisymposium on antiparasitic chemotherapy, which was a major focus of the conference.

\section{DRUGS IN CURRENT USE}

Artemisinin. Derivatives of artemisinin are used widely because they are one of the few effective treatments for multi-drug resistant Plasmodium falciparum malaria. Understanding the mechanism of artemisinin action may aid in the development of better second generation drugs. Data presented by Steve Meshnick from the University of Michigan (Figure 1) suggest that artemisinin has a two step mechanism of action. ${ }^{1-3}$ First, the drug interacts with intraparasitic heme that catalyzes its conversion into a free radical. Second, the free radical form of the drug alkylates critical malarial proteins. Evidence for the first step is based on electron spin resonance spectroscopy and cyclic voltammetry studies that demonstrate that heme can catalyze 


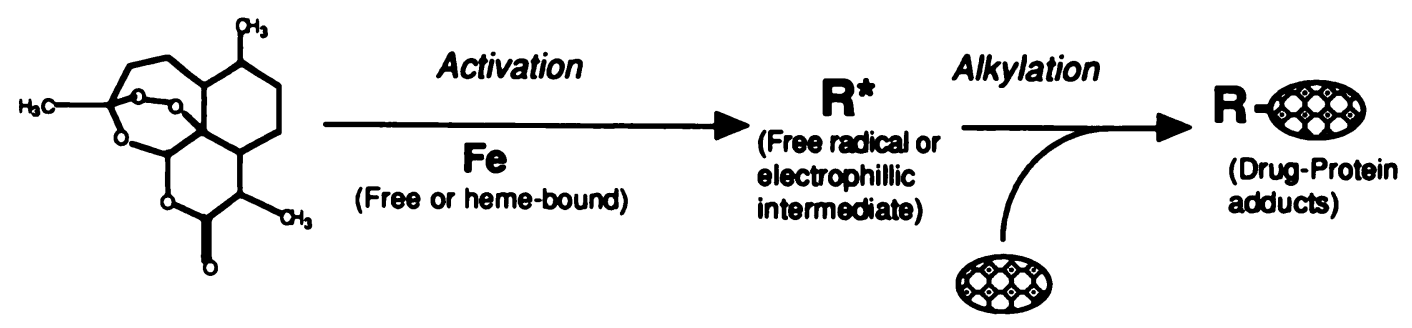

Malaria target proteins

Figure 1. Proposed two step mechanism of action of artemisinin. First, the drug interacts with intraparasitic heme that catalyzes its conversion into a free radical. Second, the free radical form of the drug alkylates critical malarial proteins.

the opening of the drug's endoperoxide bridge. The fact that iron chelators and free radical scavengers antagonize the antiplasmodial activity of artemisinin in vitro suggests that heme-induced degradation of the endoperoxide bridge may be important in vivo. Evidence for the second alkylation step is based on two observations: 1) alkylation of human serum albumin by artemisinin in vitro, and 2) sodium dodecyl sulfate gel autoradiograms that demonstrate the alkylation of parasite proteins by radiolabeled drug.

Studies presented by Mamoru Suzuki from Gunma University suggest that artemether has a rapid onset of action in vivo in $P$. falciparuminfected Aotus monkeys. ${ }^{4}$ Distinct morphologic changes were seen within $2 \mathrm{hr}$ and viable parasites disappeared within $66 \mathrm{hr}$, although dead intraerythrocytic parasites persisted in the circulation for up to 10 days. Ultrastructural studies showed marked swelling and disruption of the inner membrane of parasite mitochondria within $2 \mathrm{hr}$. Late changes included clumping of ribosomes and the appearance of electron dense material in the nucleus. Together these observations suggest that mitochondria, which contain many hemoproteins, may be an important target for artemisinin. If this hypothesis is correct, additional information will be required to explain the specificity of artemisinin, i.e., why are the artemisinin concentrations that inhibit parasite growth by damaging their mitochondria not toxic also for mammalian cells at those concentrations?

Dihydrofolate reductase (DHFR) inhibitors. Toshihiro Horii from Osaka University has used a novel approach to study the action of DHFR inhibitors against the $P$. falciparum DHFR in vitro. To circumvent problems asso- ciated with the expression of AT-rich parasite DNA in Escherichia coli, he synthesized an artificial $P$. falciparum DHFR gene in vitro using characteristic $E$. coli codons to produce the desired amino acid sequence of the parasite DHFR. ${ }^{5}$ Expression of this construct in $E$. coli yielded $\sim 30 \%$ of cell protein as the parasite DHFR. Because most of the parasite DHFR was in aggregates, it did not demonstrate enzymatic activity. However, after solubilization and renaturation, the enzymatic activity, $K_{m}$, and $V_{\max }$ of the parasite DHFR were similar to those of the purified bifunctional $P$. falciparum DHFRthymidylate synthase complex, ${ }^{6}$ and $K_{i} s$ values were similar to those obtained previously with known inhibitors. This recombinant parasite enzyme is now being used to test candidate DHFR inhibitors in vitro.

Single-dose diethylcarbamazine (DEC) versus ivermectin. In previous studies, DEC has been given in doses of 50-100 $\mathrm{mg}$ three times a day for up to 21 days, and has frequently produced severe limiting side effects. In contrast, ivermectin has been given as a single dose every 6-12 months and has had a much lower incidence of side effects. James Kazura from Case Western Reserve University reported a study from Papua New Guinea in which single doses of DEC and ivermectin were compared in persons with bancroftian filariasis. Although the initial decrease in microfilaremia was greater in the ivermectin group, the results with DEC and ivermectin were similar at 90 and 180 days. Most remarkable was the relative absence of severe side effects with single dose DEC. These results suggest that it may be possible to give DEC as a single dose without the severe side effects observed with 14-21-day regimens, and that single 

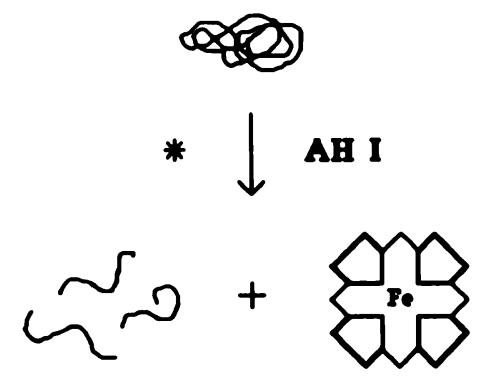

Beme
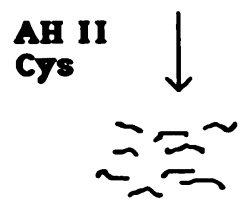

B=0

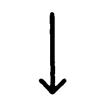

$$
\downarrow \text { HPP }
$$

Hemozoin

\section{Amino Acids}

FIGURE 2. Proposed mechanism of hemoglobin catabolism in Plasmodium falciparum. AH I and II are aspartic hemoglobinases I and II; Cys is the cysteine protease; HP is heme polymerase; Exo is a proposed exopeptidase activity. ${ }^{*}$ indicates the first enzymatic reaction.

dose DEC may produce antiparasitic effects similar to those obtained with 14-21-day courses of DEC. ${ }^{7.8}$

Effect of ivermectin on transmission of onchocerciasis. Ivermectin markedly reduces the number of microfilariae in the skin of persons with onchocerciasis. In a prospective study carried out in Guatemala, Edward Cupp from the University of Arizona reported that ivermectin also reduced the transmission of onchocerciasis. In a three-year study, four six-monthly treatments with ivermectin reduced both the prevalence and intensity of microfilarial infection in the black fly vector. ${ }^{9}$ This change was associated with an $80-100 \%$ reduction in transmission as measured by a decrease in the numbers of infective stage larvae in the Simulium ochraceum. Repetitive treatment cycles also affected adult worms, increasing the proportions of uninseminated, damaged, and dead female worms in subcutaneous nodules. ${ }^{10}$ These results are consistent with a direct drug effect on adult male and female worms in vivo.
DRUGS UNDER DEVELOPMENT OR IN CLINICAL TRIALS

Desferrioxamine (DFO). Data presented by Masamichi Aikawa and Gary Brittenham from Case Western Reserve University suggest that iron-chelating agents such as DFO may offer a new approach to the treatment of cerebral malaria by inhibiting both parasite growth and peroxidant damage to host cells. Parasite growth is inhibited by DFO in vitro, presumably because DFO chelates the iron that the parasite needs to make iron-containing enzymes such as ribonucleotide reductase. This hypothesis is also consistent with morphologic studies that demonstrated nuclear abnormalities as the earliest ultrastructural changes in parasites treated with DFO. " Results of a clinical trial indicate that DFO reduced the median duration of deep coma from 68 to $24 \mathrm{hr}$ in children with cerebral malaria, all of whom were treated with quinine, and simultaneously increased the rate of parasite clearance. $^{12}$ The variety of iron-chelating agents available raises the possibility that these agents may provide a new class of chemotherapeutic agents for severe and cerebral malaria.

\section{POTENTIALLY UNIQUE PARASITE TARGETS FOR DRUG DEVELOPMENT}

Hemoglobin degradation in Plasmodia. The intraerythrocytic malaria parasite $P$. falciparum internalizes and degrades most of its host erythrocyte's hemoglobin. Data presented by Daniel Goldberg from Washington University (Figure 2) indicate that the critical first step in hemoglobin degradation is cleavage of the $\alpha$ chain by an aspartic protease between residues 33 (Phe) and 34 (Leu). ${ }^{13}$ Examination of multiple hemoglobin sequences has shown that this cleavage site is retained in all known human hemoglobins. The gene for this enzyme has now been cloned and found to be homologous to human renin and cathepsin $D$, although it has an unusually long propiece with a hydrophobic region consistent with a membrane spanning domain. The second enzyme involved in the proteolysis of hemoglobin is an unusual cysteine protease that appears to function only after the initial aspartic protease cleavage. These results suggest that the proteolysis of hemoglobin by plasmodia is a highly ordered process, ${ }^{14}$ and that it may be possible to design specific inhibitors 
of this process that have antiplasmodial activity both in vitro and in vivo.

Topoisomerase II in trypanosomes. The mitochondrial (kinetoplastid or k) DNA of the trypansosomes that cause African sleeping sickness contains thousands of interlocked maxi- $(\sim 20$ $\mathrm{kb})$ and mini-( $\sim 1 \mathrm{~kb})$ circles that must undergo many changes during replication and transcription. These reactions are catalyzed by topoisomerases, which are inhibited by a number of drugs in current use as antitumor or antibacterial agents: etoposide, adriamycin, amsacrine, nalidixic acid, and the fluoroquinolones. Data presented by Terry Shapiro from Johns Hopkins University indicate that inhibitors of kinetoplast topoisomerase II act in trypanosomes by stabilizing a cleavable complex between the enzyme and its DNA substrate. Studies in Trypanosoma equiperdum demonstrate that etoposide increases the concentration of cleavable complexes between mitochondrial topoisomerase II and minicircle DNA in a time-dependent fashion. The cleavage sites map to positions of importance in minicircle replication. ${ }^{15,16}$ Conventional antitrypanosomal agents such as pentamidine and samorin also inhibit kinetoplast topoisomerase II activity, and may act against trypanosomes in part by this mechanism. These results support the hypothesis that mitochondrial and nuclear topoisomerase II activities are distinct; they suggest that kinetoplast topoisomerase II enzymes may be important targets in the chemotherapy of trypanosomiasis.

Thymidylate synthase in Plasmodia. Previous studies by Pradip Rathod and his colleagues at Catholic University (Washington, DC) have shown that 5-fluoroorotic acid inhibits the growth of $P$. falciparum in vitro' ${ }^{17}$ and $P$. yoelii in vivo (unpublished data). More recent studies indicate that the concentrations of 5-fluoroorotic acid necessary to inhibit clonal viability of parasites correlate well with those that inhibit thymidylate synthase activity, and thus suggest that the mechanism responsible for this effect is inhibition of the parasite thymidylate synthase. ${ }^{18}$

Ion channels in Plasmodia. Patch clamping, which has generally been used with much larger cells such as squid axons, has not been technically feasible previously for either the parasite or its host red blood cell (RBC). After removing the intervening RBC membrane and forming gigaohm seals on the small (3-5 $\mu \mathrm{m})$ parasite, Desai and his colleagues were able to study trans- port across the parasitophorous vacuole membrane (PVM), the outer of the two parasite membranes. This approach identified a $140 \mathrm{pS}$ channel on the PVM, which is permeable to both cations and anions, such as lysine ${ }^{+}$, $\mathrm{Tris}^{+}, \mathrm{Ca}^{++}$, $\mathrm{Mg}^{++}$, and glucuronate ${ }^{-}{ }^{19}$ This channel is present at high density and is open more than $98 \%$ of the time at the resting potential of the PVM. Together with a new $\mathrm{Ca}^{++}$pathway in the membrane of the $R B C,{ }^{20}$ these studies provide evidence for parasite-induced channels that are potentially unique chemotherapeutic targets. Neither channel is affected by chloroquine, and both are similar in chloroquine-susceptible and -resistant parasites.

Proteases in schistosomes. James McKerrow and his colleagues at the University of California in San Francisco have performed a series of studies on rationale drug design using computer modeling of the schistosomal serine protease that cercariae use to cross the skin and infect humans. ${ }^{21}$ Based on $x$-ray diffraction data for similar proteases that have been crystallized, computer modeling was used to create a model of the cercarial serine protease and to examine the interaction with inhibitors using computer graphics. Using this strategy, four of the $40 \mathrm{pu}$ tative inhibitors identified by computer modeling were found to have $K_{i}$ s values $<100 \mu \mathrm{M}$ and are therefore being subjected to further study. ${ }^{22}$ These studies support the idea that specific inhibitors of parasite enzymes that are effective in vitro and in vivo can be developed with the assistance of computer modeling. Additional studies with peptidomimetic inhibitors have confirmed that cysteine proteases of Schistosoma mansoni and Trypanosoma cruzi can be selectively inhibited in vitro and in vivo without toxicity at concentrations that interrupt the parasite life cycle.

Financial support: These studies were supported by NIH grants R22 AI-26848 (Steve Meshnick), P01 AI15351 (James Kazura), AI-29971 (Edward Cupp), AI10645 (Masamichi Aikawa and Gary Brittenham), R29 AI-31615 (Daniel Goldberg), AI-28855 (Terry Shapiro), AI-26912 (Pradip Rathod), R22 AI-25136 (Donald Krogstad), and AI-20452 (James McKerrow).

Authors' addresses: Tulane School of Public Health and Tropical Medicine, New Orleans, LA 70112. Mamoru Suzuki, Gunma University School of Medicine, Maebashi 371, Japan. Carole A. Long, Hahnemann University, Philadelphia, PA 19102. Yoshiki Aoki, Nagasaki University, Nagasaki 852, Japan. Akira Ishii, National Institute of Health, Tokyo 162, Japan. Ste- 
phanie L. James, Solar Building, Room 3A-02, National Institute of Allergy and Infectious Diseases, National Institutes of Health, 6003 Executive Boulevard, Bethesda, MD 20892.

\section{REFERENCES}

1. Meshnick, SR, Yang YZ, Lima V, Kuypfers F, Kamchonwongpaisan S, Yuthavong Y, 1993. Iron-dependent free radical generation from the antimalarial agent artemisinin (qinghaosu). Antimicrob Agents Chemother 37: 1108-1114.

2. Zhang F, Gosser DK Jr, Meshnick SR, 1992. Hemin-catalyzed decomposition of artemisinin (qinghaosu). Biochem Pharmacol 43: 18051809.

3. Meshnick SR, Thomas A, Ranz A, Xu CM, Pan HZ, 1991. Artemisinin (qinghaosu): the role of intracellular hemin in its mechanism of antimalarial action. Mol Biochem Parasitol 49: 181-189.

4. Kawai S, Kano S, Suzuki M, 1993. Morphologic effects of artemether on Plasmodium falciparum in Aotus trivirgatus. Am J Trop Med Hyg 49: 812-818.

5. Sano G, Morimatu K, Horii T, 1994. Purification and characterization of dihydrofolate reductase of Plasmodium falciparum expressed by a synthetic gene in Escherichia coli. Mol Biochem Parasitol 63: 265-273.

6. Chen G-X, Zolg JW, 1987. Purification of the bifunctional thymidylate synthase-dihydrofolate reductase complex from the human malaria parasite Plasmodium falciparum. Mol Pharmacol 32: 723-730.

7. Kazura JW, Greenberg J, Perry R, Weil G, Day KP, Alpers MH, 1993. Comparison of singledose diethylcarbamazine and ivermectin for treatment of bancroftian filariasis in Papua New Guinea. Am J Trop Med Hyg 49: 804-811.

8. Kazura JW, 1993. Ivermectin in human filariasis. Microb Pathog 14: 337-342.

9. Cupp EW, Ochoa JO, Collins RC, Cupp MS, Gonzales-Peralta C, Castro J, Zea-Flores G, 1992. The effects of repetitive community-wide ivermectin treatment on transmission of Onchocer ca volvulus in Guatemala. Am J Trop Med Hyg 47: 170-180.

10. Duke BO, Zea-Flores G, Castro J, Cupp EW, Munoz B, 1991. Comparison of the effects of a single dose and of four six-monthly doses of ivermectin on adult Onchocerca volvulus. Am J Trop Med Hyg 45: 132-137.

11. Atkinson CT, Bayne MT, Gordeuk VR, Brittenham GM, Aikawa M, 1991. Stage-specific ul- trastructural effects of desferrioxamine on Plasmodium falciparum in vitro. Am J Trop Med Hyg 45: 593-601.

12. Gordeuk V, Thuma P, Brittenham GM, McLaren C, Parry D, Backenstose A, Biemba G, Msiska R, Holmes L, McKinley E, Vargas L, Gilkeson R, Poltera A, 1992. Effect of iron chelation therapy on recovery from deep coma in children with cerebral malaria. $N$ Engl J Med 327: 1473-1477.

13. Goldberg DE, Slater AF, Beavis R, Chait B, Cerami A, Henderson GB, 1991. Hemoglobin degradation in the human malaria pathogen Plasmodium falciparum: a catabolic pathway initiated by a specific aspartic protease. J Exp Med 173: 961-969.

14. Goldberg DE, Slater AF, Cerami A, Henderson GB, 1990. Hemoglobin degradation in the malaria parasite Plasmodium falciparum: an ordered process in a unique organelle. Proc Natl Acad Sci USA 87: 2931-2935.

15. Shapiro TA, Klein VA, Englund PT, 1989. Drugpromoted cleavage of kinetoplast DNA minicircles: evidence for type II topoisomerase activity in trypanosome mitochondria. J Biol Chem 264: 4173-4178.

16. Shapiro TA, England PT, 1990. Selective cleavage of kinetoplast DNA minicircles promoted by antitrypanosomal drugs. Proc Natl Acad Sci USA 87: 950-954.

17. Young RD, Rathod PK, 1993. Clonal viability measurements on Plasmodium falciparum to assess in vitro schizonticidal activity of leupeptin, chloroquine and 5-fluoroorotate. Antimicrob Agents Chemother 37: 1102-1107.

18. Rathod PK, Leffers NP, Young RD, 1992. Molecular targets of 5-fluoroorotate in the human malaria parasite, Plasmodium falciparum. Antimicrob Agents Chemother 35: 704-711.

19. Desai SA, Krogstad DJ, McCleskey EW, 1993. A nutrient-permeable channel on the intraerythrocytic malaria parasite. Nature 362: 643-646.

20. Desai SA, Schlesinger PH, Krogstad DJ, 1991. Physiologic rate of $\mathrm{Ca}^{2+}$ entry matches active extrusion in human erythrocytes. $J \operatorname{Exp}$ Med 98: 349-364.

21. Cohen FE, Gregoret LM, Amiri P, Aldape K, Railey J, McKerrow JH, 1991. Arresting tissue invasion of a parasite by protease inhibitors chosen with the aid of computer modeling. Biochemistry 30: 11221-11229.

22. Ring CS, Sun E, McKerrow JH, Lee GK, Rosenthal PJ, Kuntz ID, Cohen FE, 1993. Structurebased inhibitor design by using protein models for the development of antiparasitic agents. Proc Natl Acad Sci USA 90: 3583-3587. 